\title{
Human caspase-1 autoproteolysis is required for ASC-dependent and -independent inflammasome activation
}

Daniel P. Ball ${ }^{1 \#}$, Cornelius Y. Taabazuing ${ }^{1 \#}$, Andrew R. Griswold ${ }^{2}$, Elizabeth L. Orth ${ }^{3}$, Sahana D. $\mathrm{Rao}^{3}$, Ilana B. Kotliar ${ }^{3}$, Darren C. Johnson ${ }^{3} \&$ Daniel A. Bachovchin ${ }^{1,3,4^{*}}$

\author{
Affiliations: \\ ${ }^{1}$ Chemical Biology Program, Memorial Sloan Kettering Cancer Center, New York, New York \\ 10065, USA. \\ ${ }^{2}$ Weill Cornell/Rockefeller/Sloan Kettering Tri-Institutional MD-PhD Program, New York, New \\ York 10065, USA. \\ 3 Tri-Institutional PhD Program in Chemical Biology, Memorial Sloan Kettering Cancer Center, \\ New York, New York 10065, USA. \\ ${ }^{4}$ Pharmacology Program of the Weill Cornell Graduate School of Medical Sciences, Memorial \\ Sloan Kettering Cancer Center, New York, New York 10065, USA.
}

${ }^{*}$ Correspondence to Daniel A. Bachovchin: bachovcd@mskcc.org

${ }^{\#}$ D.P. Ball and C.Y. Taabazuing contributed equally to this paper. 


\section{Abstract}

2 Pathogen-related signals induce a number of cytosolic pattern-recognition receptors (PRRs) to

3 form canonical inflammasomes, which activate pro-caspase-1 and trigger pyroptotic cell death.

4 All well-studied PRRs oligomerize with the pro-caspase-1-adapter protein ASC to generate a

5 single large structure in the cytosol, which induces the autoproteolysis and activation of the pro-

6 caspase-1 zymogen. However, several PRRs can also directly interact with pro-caspase-1

7 without ASC, forming much smaller "ASC-independent" inflammasomes. It is currently thought

8 that pro-caspase-1 autoproteolysis does not occur during, and is not required for, ASC-

9 independent inflammasome activation. Here, we show that the related human PRRs NLRP1 and

10 CARD8 exclusively form ASC-dependent and ASC-independent inflammasomes, respectively,

11 identifying CARD8 as the first PRR that cannot form an ASC-containing signaling platform.

12 Despite their different structures, we discovered that both the NLRP1 and CARD8 inflammasomes

13 require pro-caspase-1 autoproteolysis between the small and large catalytic subunits to induce

14 pyroptosis. Thus, pro-caspase-1 self-cleavage is an obligate regulatory step in the activation of

15 human canonical inflammasomes. 


\section{Introduction}

Caspase- 1 is a cysteine protease that induces pyroptotic cell death in response to a number of pathogen-associated signals (Broz and Dixit, 2016; Lamkanfi and Dixit, 2014). Typically, an intracellular pattern recognition receptor (PRR) senses a particular microbial

31 structure or activity and oligomerizes with the adapter protein ASC to form an "ASC focus" in the

32 cytosol (Broz et al., 2010a; Jones et al., 2010). The pro-caspase-1 zymogen is recruited to this

33 structure, where it undergoes proximity-induced autoproteolysis to generate a catalytically-active

34 enzyme. Mature caspase-1 then cleaves and activates the inflammatory cytokines pro-IL-1 $1 \beta$ and

35 pro-IL-18 and the pore-forming protein gasdermin D (GSDMD), causing inflammatory cell death

36 (Kayagaki et al., 2015; Shi et al., 2015). Collectively, the structures that activate pro-caspase-1

37 are called "canonical inflammasomes".

Two death-fold domains, the pyrin domain (PYD) and the caspase activation and recruitment domain (CARD), mediate canonical inflammasome assembly (Broz and Dixit, 2016). ASC is comprised of a PYD and a CARD (Fig. 1A), and bridges either a PYD or a CARD of an

41 activated PRR to the CARD of pro-caspase-1. In mice, all known pro-caspase-1-activating PRRs

42 form ASC-containing inflammasomes. However, in the absence of ASC, two murine CARD-

43 containing PRRs, NLRC4 and NLRP1B, can directly recruit and activate pro-caspase-1 through

44 CARD-CARD interactions (Broz et al., 2010b; Guey et al., 2014; Mariathasan et al., 2004; Poyet

45 et al., 2001; Van Opdenbosch et al., 2014). ASC-independent inflammasomes induce the

46 cleavage of GSDMD and trigger lytic cell death, but do not form large foci or efficiently process

47 pro-caspase-1 and pro-IL-1 $\beta$ (Broz et al., 2010b; He et al., 2015).

These observations suggested that pro-caspase-1 autoproteolysis may not be required

49 for cell death. To explore this possibility, two independent groups reconstituted Casp $1^{\text {-/ }}$ mouse macrophages (which expressed ASC) with an uncleavable mutant form of mouse pro-caspase-1,

51 and found that the mutant enzyme still mediated cell death, but did not process pro-IL-1 $\beta$, in 
52 response to various inflammasome stimuli (Broz et al., 2010b; Guey et al., 2014). Another study,

53 performed after the discovery of GSDMD, showed that the uncleavable mutant pro-caspase-1

54 was partially defective in processing GSDMD and inducing pyroptosis in ASC-expressing RAW

55264.7 cells in response to NLRP3 inflammasome activation (He et al., 2015). Taken together,

56 these studies indicated that murine ASC-containing inflammasomes can activate pro-caspase-1

57 to some extent, but that autoproteolysis was required for full catalytic activity. Because ASC-

58 independent inflammasomes induce little detectable pro-caspase-1 and pro-IL-1 $\beta$ processing, it

59 has been widely assumed that ASC-independent inflammasomes specifically activate the pro-

60 protein form of caspase-1 without autoproteolysis. However, the importance of pro-caspase-1

61 autoproteolysis in mouse ASC-independent inflammasome activation has not been directly

62 tested, perhaps in part because these structures are not known to form in physiologically-relevant

63 macrophages that express ASC. Moreover, the requirement of human pro-caspase-1

64 autoproteolysis in the activation of either ASC-independent or ASC-dependent inflammasomes

65 has not been evaluated experimentally.

DPP8/9 inhibitors activate the related CARD-containing human NLRP1 and CARD8

67 inflammasomes (Fig. 1A), which both have C-terminal ZU5, UPA, and CARD domains (Chui et

68 al., 2019; Johnson et al., 2018; Okondo et al., 2017; Zhong et al., 2018). The ZU5 domains of

69 NLRP1 and CARD8 undergo post-translational autoproteolysis (Fig. 1A), generating non-

70 covalently associated, autoinhibited $\mathrm{N}$ - and C-terminal polypeptide fragments (D'Osualdo et al.,

71 2011; Finger et al., 2012; Frew et al., 2012). The C-terminal UPA-CARD fragments mediate cell

72 death (Finger et al., 2012; Johnson et al., 2018). CARD8 does not require ASC to activate pro-

73 caspase-1 (Johnson et al., 2018; Okondo et al., 2017), but it is unknown whether CARD8 can

74 also form an ASC-containing inflammasome. In contrast, human NLRP1, unlike mouse NLRP1A

75 and NLRP1B (Masters et al., 2012; Van Opdenbosch et al., 2014), appears to require ASC (Finger

76 et al., 2012; Zhong et al., 2016; Zhong et al., 2018). Here, we show that CARD8 and NLRP1 
77 exclusively form ASC-independent and ASC-dependent inflammasomes, respectively, due to

78 specific CARD-CARD interactions. These data identify CARD8 as the first pro-caspase-179 activating PRR that cannot form an ASC focus. Although the CARD8 inflammasome induces little

80 detectable pro-caspase-1 processing by immunoblotting (Johnson et al., 2018; Okondo et al., 81 2017), we found that pro-caspase-1 autoproteolysis was required for activation of both the CARD8

82 and NLRP1 inflammasomes. Overall, these data demonstrate that autoproteolysis is critical for

83 the activation of human canonical inflammasomes.

85 Results and discussion

86 NLRP1 is ASC-dependent and CARD8 is ASC-independent

87 We first wanted to determine the capabilities of human NLRP1 and CARD8 to form ASC88 dependent and ASC-independent inflammasomes. We therefore transfected constructs encoding 89 NLRP1, CARD8, and/or ASC into HEK 293T cells stably expressing pro-caspase-1 and GSDMD 90 before treatment with the DPP8/9 inhibitor Val-boroPro (VbP). VbP induced similar levels of

91 GSDMD cleavage and LDH release in cells expressing CARD8 in the presence or absence of

92 ASC (Fig. 1B,C), confirming that ASC is not required for CARD8-mediated cell death (Johnson

93 et al., 2018; Okondo et al., 2017). In contrast, NLRP1 required ASC co-expression to mediate 94 cell death (Fig. 1B,C). We should note that the co-expression of NLRP1 and ASC induced some 95 spontaneous cell death and GSDMD cleavage, but both were increased by VbP. Consistent with 96 these data, transient transfection of constructs encoding the active UPA-CARD fragment of 97 NLRP1, but not CARD8, required ASC to induce GSDMD cleavage (Fig. S1A). As previously 98 reported, the PYD of NLRP1 was dispensable for inflammasome activation (Fig. S1B,C) 99 (Chavarria-Smith et al., 2016; Finger et al., 2012).

100 Although these results confirm that CARD8 can directly activate pro-caspase- 1 without 101 ASC bridging, it remained possible that CARD8 could also form an ASC-containing 
102 inflammasome, similar to mouse NLRP1B (Van Opdenbosch et al., 2014). We next co-

103 transfected HEK 293T cells with constructs encoding GFP-tagged ASC and either NLRP1 or

$104 \mathrm{CARD} 8$. These cells were then treated with $\mathrm{VbP}$ for $6 \mathrm{~h}$ and imaged by fluorescence microscopy

105 (Fig. 1D,E). VbP induced ASC specks in NLRP1, but not CARD8, expressing cells, suggesting

106 that CARD8 cannot form an ASC-containing inflammasome. Similarly, transfection of the UPA-

107 CARD of NLRP1, but not CARD8, induced ASC speck formation (Fig. S1D,E). To further support

108 these microscopy results, we co-transfected HEK 293T cells with constructs encoding untagged

109 ASC and either NLRP1 or CARD8, treated the cells with VbP, and cross-linked lysates with

110 disuccinimidyl suberate (DSS). As expected, VbP induced ASC oligomerization in cells

111 expressing NLRP1, but not CARD8 (Fig. 1F).

112 We hypothesized that the exclusive formation of ASC-independent and ASC-dependent

113 inflammasomes by CARD8 and NLRP1, respectively, was due to specific interaction differences

114 between the CARDs of CARD8 and NLRP1 with the CARDs of ASC and CASP1. To test this

115 prediction, we incorporated these CARDs into a split luciferase-based NanoBiT assay (Dixon et

116 al., 2016), fusing Small BiT (SmBiT, an 11 amino acid peptide) to the CARD domains of ASC and

117 CASP1 and Large BiT (LgBiT, an $18 \mathrm{kDa}$ tag that luminesces only when bound to SmBiT) to the

118 CARD domains of ASC, CASP1, CARD8, and NLRP1 (Fig. 2A). We mixed lysates containing

119 the indicated fusion proteins, and observed luminescent signals indicating binding between the 120 ASC ${ }^{\text {CARD }}$ and itself, CASP1 ${ }^{\text {CARD }}$, and NLRP1 ${ }^{\text {CARD }}$ (Fig. 2B), and between the CASP $1^{\text {CARD }}$ and itself, 121 ASC ${ }^{\text {CARD }}$, and CARD8 ${ }^{\text {CARD }}$ (Fig. 2C). As expected, we did not observe a CASP1 ${ }^{\text {CARD }}-$ NLRP1 ${ }^{\text {CARD }}$ 122 interaction or an ASC ${ }^{\text {CARD }}$-CARD8 ${ }^{\text {CARD }}$ interaction. Overall, these results indicate specific CARD123 CARD interactions govern the formation of the CARD8 ASC-independent inflammasome and the 124 NLRP1 ASC-dependent inflammasome.

125

126 Proteasome activity is critical for NLRP1 activation 
DPP8/9 inhibition induces the proteasome-mediated degradation of the $\mathrm{N}$-terminal

128 fragment of mouse NLRP1B and CARD8, releasing the UPA-CARD C-terminal fragment to 129 activate pro-caspase-1 (Chui et al., 2019; Johnson et al., 2018). Given the differences in the N130 terminal regions of CARD8 and NLRP1 (Fig. 1A), we wanted to confirm that VbP activates human 131 NLRP1 by a similar degradation mechanism. Indeed, autoproteolysis-defective NLRP1 S1213A, 132 which is unable to release its C-terminal fragment, was severely impaired in VbP-induced ASC 133 speck formation (Fig. S2A,B) and cell death (Fig. S2C,D). Moreover, proteasome inhibitors 134 partially rescued VbP-induced cell death (Fig. 3A, Fig. S1B), GSDMD cleavage (Fig. 3B, Fig. 135 S1C), and ASC oligomerization (Fig. 3C). We speculate that proteasome blockade did not fully 136 rescue $\mathrm{VbP}$-induced NLRP1 activation because very small amounts of UPA-CARD are needed 137 to nucleate ASC specks (Sandstrom et al., 2019), and therefore even slight residual proteasome 138 activity could be sufficient to activate the inflammasome. Consistent with only a small amount of 139 NLRP1 UPA-CARD being liberated, VbP did not induce obvious NLRP1 protein depletion by 140 immunoblotting in several experiments (Fig. 1C, Fig. 3B). However, we confirmed that VbP does 141 indeed induce NLRP1 protein depletion by treating NLRP1-expressing HEK 293T cells with VbP 142 for longer time periods (Fig. S2E). It should be noted that these cells do not express pro-caspase1431 , and thus NLRP1 loss here is not due to selective elimination of NLRP1-expressing cells.

144 Germline mutations in the N-terminal fragment of NLRP1 cause several related 145 inflammatory skin disorders (Zhong et al., 2016; Zhong et al., 2018). We hypothesized that these 146 mutations destabilized the $\mathrm{N}$-terminal fragment, leading to increased proteasome-mediated $\mathrm{N}$ 147 terminal degradation. Indeed, we found that the proteasome inhibitor bortezomib reduced 148 spontaneous inflammasome activation caused by several of these mutations (Fig. S2F,G). 149 Overall, these data indicate that the proteasome mediates both $\mathrm{VbP}$ - and mutation-induced 150 NLRP1 activation. 
154 in greater detail. We initially discovered DPP8/9 inhibitor-induced pyroptosis in human THP-1

155 cells (Okondo et al., 2017), which is mediated by CARD8 (Johnson et al., 2018). We observed

156 little, if any, caspase-1 and IL-1 $\beta$ processing, and designated this death as "pro-caspase-1

157 dependent" pyroptosis. However, as described above, we never formally demonstrated that pro-

158 caspase-1 itself mediates this response. We reasoned that we might observe more caspase-1

159 processing, if it was occurring, in GSDMD ${ }^{-/}$THP-1 cells, as the cleaved products would not be as

160 readily released into the supernatant. Indeed, we did observe bands corresponding to the p35

161 and p20 fragments in these knockout cells (Fig. 4A-C), indicating that the CARD8 inflammasome

162 can, in fact, process pro-caspase-1. It should be noted that VbP induces apoptosis in $G S D M D^{-/-}$

163 THP-1 cells (Taabazuing et al., 2017), and as expected PARP cleavage was observed here.

We next wanted to determine if caspase-1 processing was required for cell death. Analogous to the previously created uncleavable mouse pro-caspase-1 (mCASP1 D6N) (Broz et al., 2010b), we generated an uncleavable human pro-caspase-1 (CASP1 D5N, Fig. 4C) in which all Asp cleavage sites were mutated to Asn residues (Thornberry et al., 1992). We then created 168 HEK 293T cell lines stably expressing wild-type (WT), uncleavable (D5N), or catalytically-inactive 169 (C285A) pro-caspase-1, transiently transfected constructs encoding WT or autoproteolytic170 defective S297A CARD8 into each these cell lines, and treated with VbP. As expected, we 171 observed robust cell death and GSDMD cleavage in cells with WT pro-caspase-1 and WT 172 CARD8, but not in cells expressing catalytically-dead CASP1 or autoproteolysis-defective CARD8

173 (Fig. 4D). Interestingly, we also observed a small amount of the p20 cleaved product in the cell 174 line expressing CASP1 WT. In contrast, we did not observe any cell death or GSDMD cleavage 175 in cells expressing the uncleavable CASP1 D5N. Consistent with these data, transient 176 transfection of a plasmid encoding the active UPA-CARD fragment of CARD8 did not induce 
GSDMD cleavage in cells expressing CASP1 D5N (Fig. S3A). Together, these data indicate that pro-caspase-1 autoproteolysis is required for CARD8 inflammasome activation.

\section{Cleavage in the caspase-1 interdomain linker (IDL) is essential for activation}

We next wanted to determine which specific pro-caspase-1 cleavage sites were required for CARD8 inflammasome activation, and to determine if pro-caspase-1 autoproteolysis was also required for NLRP1 inflammasome activation. Pro-caspase-1 is comprised of three domains, a

184 CARD, a large subunit (LS, p20), and a small subunit (SS, P10), separated by two linkers (Fig.

185 4C). Pro-caspase-1 undergoes proteolytic processing at two sites (D103 and D119) in the CARD

186 linker (CDL) that separates the CARD and the p20, and three sites (D297, D315, and D316) in

187 the interdomain linker (IDL) that separates the p20 and the p10 (Boucher et al., 2018; Thornberry

188 et al., 1992). As IDL cleavage has been associated with higher catalytic activity and CDL cleavage

189 with termination of activity (Boucher et al., 2018; Broz et al., 2010b), we first tested the 3 putative

190 cleavage sites in the IDL by generating HEK 293T cells stably expressing CASP1 D297N,

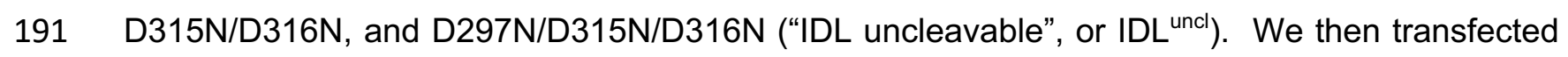

192 plasmids encoding CARD8 or both NLRP1 and ASC into these cell lines and treated with VbP.

193 We found that VbP induced LDH release and GSDMD cleavage in cells expressing CASP1

194 D297N and CASP1 D315N/D315N, but not in cells expressing CASP1 IDL ${ }^{\text {uncl }}$ (Fig. 5). These data

195 show that pro-caspase-1 autoproteolysis within the IDL is critical for both ASC-independent and

196 -dependent inflammasome activation. As predicted by these results, transfection of plasmids

197 encoding ASC, the UPA-CARD of CARD8, or residues 1-328 of human NLRC4, which contains

198 a CARD domain that can directly activate human CASP1 (Poyet et al., 2001), failed to induce

199 death in the cells expressing CASP1 IDL ${ }^{\text {uncl }}$ (Fig. S3B-D). In contrast to human CASP1 D5N and

200 consistent with previous reports (Broz et al., 2010b; Guey et al., 2014), the UPA-CARD of mouse

201 NLRP1B induced cell death in HEK 293T cells stably expressing the mouse CASP1 D6N protein 
202 (Fig. S3E,F). Surprisingly, however, the NLRP1B UPA-CARD also induced the formation of 203 several lower molecular weight caspase-1 species in the CASP1 D6N-expressing line, indicating 204 that the mouse CASP1 D6N protein is, in fact, cleavable. These potential additional cleavage 205 sites and their function in mouse caspase-1 activation warrant future investigations.

Replacing CARDs with DmrB domains enables the small-molecule (AP-20187)-induced dimerization and activation of caspases (Boucher et al., 2018; Ross et al., 2018; Ruhl et al., 2018). To confirm that IDL cleavage was required for proximity-induced pro-caspase-1 activation, we

209 cloned DmrB-caspase-1 constructs with the IDL mutations described above (Fig. S3G). We 210 transiently transfected these constructs into HEK 293T cells, and then treated these cells with the 211 AP-20187. We observed that the WT DmrB-caspase-1 underwent significant autoproteolysis and

212 triggered GSDMD cleavage (Fig. S3H). Some pro-caspase-1 autoproteolysis and GSDMD 213 cleavage were also observed for D297N and the D315N/D316N mutants, but not the IDL ${ }^{\text {uncl }}$ 214 mutant. Thus, these data confirm the importance of IDL processing for human caspase-1 215 activation.

Here, we have shown that the related NLRP1 and CARD8 inflammasomes are remarkably

217 distinct. First, these PRRs have functionally divergent C-terminal UPA-CARD fragments - one 218 that induces an ASC focus to indirectly activate pro-caspase-1 and one that directly activates pro219 caspase-1. As such, we predict that the physiological outputs of NLRP1 and CARD8 activation 220 will be different in vivo, for example in the kinetics of immune activation or in the type or extent of 221 cytokine processing. Future investigations are needed to establish the biological purpose of ASC222 independent and ASC-dependent inflammasomes. Second, CARD8 and NLRP1 have entirely 223 dissimilar N-terminal fragments. Although both are activated by at least one similar signal-the 224 cellular consequence of DPP8/9 inhibition-we speculate that these $\mathrm{N}$-terminal fragments likely 225 evolved for different purposes that remain to be elucidated. 
More generally, we have now demonstrated that human pro-caspase- 1 autoproteolysis is necessary for both ASC-dependent and ASC-independent inflammasome activation.

228 Interestingly, two recent studies have demonstrated that the related inflammatory caspase-11, 229 which only forms an ASC-independent inflammasome (termed the "non-canonical" 230 inflammasome) with often little detectable self-cleavage and no IL-1 $\beta$ processing (Hagar et al., 231 2013; Yang et al., 2015), also requires IDL autoproteolysis for activation (Boucher et al., 2018;

232 Lee et al., 2018). In this way, the ASC-independent caspase-1 canonical inflammasome is 233 remarkably similar to the non-canonical caspase-11 inflammasome. Collectively, these reports 234 and our data show that limited proteolysis plays a critical role in the activation of inflammatory 235 caspases.

\section{Materials and Methods}

\section{Antibodies and reagents}

239 Antibodies used include: GSDMD Rabbit polyclonal Ab (Novus Biologicals, NBP2-33422), human 240 NLRP1/NALP1 Sheep polyclonal antibody (R\&D systems, AF6788), V5 Rabbit polyclonal Ab 241 (Abcam, Ab9116), FLAG® M2 monoclonal Ab (Sigma, F3165), CARD8 N-terminus Rabbit 242 polyclonal antibody (Abcam, Ab194585), CARD8 C-terminus Rabbit polyclonal Ab (Abcam, 243 Ab24186), human ASC Sheep polyclonal antibody (R\&D systems, AF3805), GAPDH Rabbit 244 monoclonal Ab (Cell Signaling Tech, 14C10), NLuc (Lg-BiT) polyclonal antibody (courtesy of 245 Promega), human Caspase-1 p20 Rabbit polyclonal Ab (Cell Signaling Technology, \#2225), 246 PARP Rabbit polyclonal Ab (Cell Signaling Technology, \#9542), IRDye 680 RD Streptavidin, (LI247 COR 926-68079), IRDye 800CW anti-rabbit (LICOR, 925-32211), IRDye 800CW anti-mouse (LI248 COR, 925-32210), IRDye 680CW anti-rabbit (LI-COR, 925-68073), IRDye 680CW anti-mouse 249 (LI-COR, 925-68072). Other reagents used include: Val-boroPro (VbP)(Okondo et al., 2017), 250 Bortezomib (MilliporeSigma, 504314), MG132 (MilliporeSigma, 474790), Carfilzomib (Cayman 
251 Chemical, 17554), B/B Homodimerizer (Takara, 635059, equivalent to AP-20187), disuccinimidyl

252 suberate (DSS, ThermoFisher Scientific, 21655), FuGENE HD (Promega, E2311).

\section{Cell Culture}

255 HEK 293T cells and THP-1 cells were purchased from ATCC. HEK 293T cells were grown in

256 Dulbecco's Modified Eagle's Medium (DMEM) with L-glutamine and 10\% fetal bovine serum

257 (FBS). THP-1 cells were grown in Roswell Park Memorial Institute (RPMI) medium 1640 with

258 L-glutamine and $10 \%$ fetal bovine serum (FBS). All cells were grown at $37{ }^{\circ} \mathrm{C}$ in a $5 \% \mathrm{CO}_{2}$

259 atmosphere incubator. Cell lines were regularly tested for mycoplasma using the MycoAlert ${ }^{\mathrm{TM}}$

260 Mycoplasma Detection Kit (Lonza). Stable cell lines were generated as described previously

261 (Johnson et al., 2018).

262

263 Cloning

264 Plasmids for full-length and truncated CARD8, NLRP1, mouse NLRP1B (allele 1), mouse and 265 human GSDMD, and mouse and human CASP1 (Johnson et al., 2018; Okondo et al.; Okondo 266 et al., 2018) were cloned as previously described and shuttled into modified pLEX_307 vectors 267 (Addgene) using Gateway technology (Thermo Fisher Scientific). A plasmid encoding NLRC4 268 was purchased from Origene (RC206757) and cloned into the Gateway system. A pLEX_307 269 vector containing RFP was used for controls. Point mutations were generated using the 270 QuickChange II site-directed mutagenesis kit (Agilent, 200523) following the manufacturer's 271 instructions. The NLRP1 $\triangle$ PYD construct starts at Ser93. DNA encoding SmBit and LgBit for 272 the NanoBiT assay (Promega) were inserted after the attR2 recombination site in a modified 273 pLEX_307 vector (immediately after the EcoRV site), and DNA encoding CARD domains were 274 shuttled into these modified vectors using Gateway technology. DmrB $\Delta$ CARD caspase-1 275 chimera constructs were cloned using assembly PCR reactions beginning at Asp92 of 276 caspase-1. 


\section{Transient transfections}

279 HEK 293T cells were plated in 6-well culture plates at $5.0 \times 10^{5}$ cells/well in DMEM. The next day, 280 the indicated plasmids were mixed with an empty vector to a total of $2.0 \mu \mathrm{g}$ DNA in $125 \mu$ in Opti281 MEM and transfected using FuGENE HD (Promega) according to the manufacturer's protocol. 282 Unless indicated otherwise, $0.02 \mu \mathrm{g}$ CARD8, $0.02 \mu \mathrm{g}$ NLRP1, and $0.005 \mu \mathrm{g}$ ASC were used. The 283 next day, the cells were treated as described. For microscopy experiments, cells were plated 284 directly into Nunc Lab-Tek II Chamber slide w/Cover sterile glass slides (Thermo Fisher Scientific, $285154534)$ at $8.0 \times 10^{4}$ cells/well and treated with $25 \mu \mathrm{L}$ transfection master mix dropwise.

\section{LDH cytotoxicity and immunoblotting assays}

288 HEK 293T cells were transiently transfected and inhibitor treated as indicated. THP-1 cells were 289 plated in 6-well culture plates at $5.0 \times 10^{5}$ cells/well and treated with $\mathrm{VbP}$ as indicated. $15 \mathrm{~min}$ 290 prior to the conclusion of cell transfection experiments $80 \mu \mathrm{L}$ of a $9 \%$ Triton $\mathrm{X}-100$ solution was 291 added to designated lysis control wells of a 6-well culture plate to completely lyse the cell contents.

292 Supernatants were analyzed for LDH activity using the Pierce LDH Cytotoxicity Assay Kit (Life 293 Technologies) and lysates protein content was evaluated by immunoblotting. Cells were washed $2942 \times$ in PBS $(\mathrm{pH}=7.4)$, resuspended in PBS, and lysed by sonication. Protein concentrations were 295 determined using the DCA Protein Assay kit (Bio-Rad). The samples were separated by SDS296 PAGE, immunoblotted, and visualized using the Odyssey Imaging System (Li-Cor).

298 Fluorescence microscopy

299 Imaging was performed on a Zeiss Axio Observer.Z1 inverted widefield microscope using $30040 x / 0.95 N A$ air objective. Cells were plated on LabTek 8-well chambered cover glass with \#1 301 coverslip. For each chamber, 10 positions were imaged with brightfield, red, and green 
fluorescence channels as a single time point at the conclusion of the given experiment. Data was exported as raw .czi files and analyzed using custom macro written in ImageJ/FIJI. Total cell area was estimated from RFP-positive signal and the number of GFP-ASC specks were quantified using the "Analyze particles" function following threshold adjustment in the GFP positive images.

\section{Split luciferase assay}

HEK cells were seeded at $3.0 \times 10^{6}$ cells in $10 \mathrm{~cm}$ dishes and transfected with $3 \mu \mathrm{g}$ of the indicated DNA construct using FuGENE HD (Promega). 24 h post-transfection, cells were washed with cold PBS (Corning), harvested by scraping, and pelleted at $450 \times g$ for $3 \mathrm{~min}$. The pellets were resuspended in $500 \mu \mathrm{L}$ PBS and lysed by sonication. Lysates were clarified to remove bulk

312 cellular debris by centrifugation at $1000 \times g$ for $5 \mathrm{~min}$, and relative expression was normalized by

313 gel densitometry of immunoblots (Image J 1.52n software). NanoBiT assays were carried out in

314 quadruplicate in white, clear, flat-bottom, 384-well assay plates (Corning, 3765). Equal volume

315 aliquots of the corresponding SmBiT/LgBiT pairs were combined within each well from normalized

316 lysates, followed by addition of Nano-Glo Live Cell Reagent, prepared as per manufacturer's

317 instructions. Following thermal equilibration, luminescence was read on a Cytation 5 multi-modal 318 plate reader.

\section{DSS Cross-linking}

321 HEK 293T cells were treated as indicated before lysates were harvested and pelleted at $400 \times \mathrm{g}$

$3224{ }^{\circ} \mathrm{C}$ for 3 min and washed with cold PBS. Cell pellets were lysed with $200 \mu \mathrm{L} 0.5 \%$ NP-40 in TBS

323 for $30 \mathrm{~min}$ on ice in $1.75 \mathrm{~mL}$ microcentrifuge tubes. The lysates were spun down at $1,000 \mathrm{~g} 4{ }^{\circ} \mathrm{C}$

324 for 10 min to remove bulk cell debris (100 $\mu \mathrm{L}$ of supernatant was reserved for immunoblot). The

325 remaining lysate was placed in the centrifuge for $10 \mathrm{~min}$ at $20,000 \times \mathrm{g} 4{ }^{\circ} \mathrm{C}$. The obtained pellet

326 was then washed with $100 \mu \mathrm{L}$ CHAPS buffer (50 mM HEPES pH 7.5, 5 mM MgCl2, 0.5 mM EGTA, 
327 and $0.1 \% \mathrm{w} / \mathrm{v}$ CHAPS) then resuspended in $48 \mu \mathrm{L}$ CHAPS buffer. $2 \mu \mathrm{L}$ of $250 \mathrm{mM}$ DSS was

328 added and the samples were agitated at $37^{\circ} \mathrm{C}$ on a rotating orbital platform set to $1,000 \mathrm{rpm}$ for

$32945 \mathrm{~min}$ to facilitate protein cross-linking. The samples were then combined with an equal volume

330 of $2 \times$ loading dye and heated to $98^{\circ} \mathrm{C}$ for $10 \mathrm{~min}$ and prepared for immunoblot analysis.

331

332 Data analysis and statistics

333 Statistical analysis was performed using GraphPad Prism 7.0 software. Statistical significance 334 was determined using two-sided Students $t$-tests.

\section{Supplemental material}

337 Fig. S1 shows additional data related to Fig. 1 and Fig. 2, demonstrating that the UPA-CARD of 338 NLRP1, but not CARD8, interacts with ASC and requires ASC to activate pro-caspase-1. Fig. S2 339 displays additional data related to Fig. 3, confirming that the proteasome plays an important role 340 in the activation of human NLRP1. In particular, this figure shows NLRP1 autoproteolysis is 341 required, that $\mathrm{VbP}$ induces NLRP1 protein loss, and spontaneous activation of NLRP1 by 342 germline mutations is blocked by bortezomib. Fig. S3 shows data related to Fig. 4 and Fig 5., 343 confirming that human caspase-1 autoproteolysis within the IDL is required for inflammasome 344 activation.

\section{Author Contributions}

347 D.P.B, C.Y.T., A.R.G, S.D.R., and D.C.J. performed experiments. D.P.B, C.Y.T., and D.A.B. 348 designed experiments, analyzed data, and wrote the paper. I.B.K. and E.L.O. developed and 349 performed the split luciferase assay.

350

\section{Acknowledgements}


352

We thank K. Schroder and P. Broz for sharing DmrB constructs. This work was supported by the Josie Robertson Foundation (D.A.B.), a Stand Up to Cancer-Innovative Research Grant (Grant Number SU2C-AACR-IRG11-17 to D.A.B.; Stand Up to Cancer is a program of the Entertainment Industry Foundation. Research Grants are administered by the American Association for Cancer Research, the scientific partner of SU2C), the Pew Charitable Trusts (D.A.B. is a Pew-Stewart Scholar in Cancer Research), the Pershing Square Sohn Cancer Research Alliance (D.A.B.), the NIH (R01 Al137168 to D.A.B.; T32 GM007739-Andersen to A.R.G; the MSKCC Core Grant P30 CA008748), an Alfred P. Sloan Foundation Research Fellowship (D.A.B.), Gabrielle's Angel Foundation (D.A.B.), and the American Cancer Society (Postdoctoral Fellowship PF-17-224-01 CCG to C.Y.T.).

\section{References}

Boucher, D., M. Monteleone, R.C. Coll, K.W. Chen, C.M. Ross, J.L. Teo, G.A. Gomez, C.L. Holley, D. Bierschenk, K.J. Stacey, A.S. Yap, J.S. Bezbradica, and K. Schroder. 2018. Caspase-1 selfcleavage is an intrinsic mechanism to terminate inflammasome activity. J. Exp. Med. 215:827-840.

Broz, P., and V.M. Dixit. 2016. Inflammasomes: mechanism of assembly, regulation and signalling. Nat. Rev. Immunol. 16:407-420.

Broz, P., K. Newton, M. Lamkanfi, S. Mariathasan, V.M. Dixit, and D.M. Monack. 2010a. Redundant roles for inflammasome receptors NLRP3 and NLRC4 in host defense against Salmonella. J. Exp. Med. 207:1745-1755.

Broz, P., J. von Moltke, J.W. Jones, R.E. Vance, and D.M. Monack. 2010b. Differential requirement for Caspase-1 autoproteolysis in pathogen-induced cell death and cytokine processing. Cell Host Microbe 8:471-483.

Chavarria-Smith, J., P.S. Mitchell, A.M. Ho, M.D. Daugherty, and R.E. Vance. 2016. Functional and Evolutionary Analyses Identify Proteolysis as a General Mechanism for NLRP1 Inflammasome Activation. PLoS Pathog. 12:e1006052.

Chui, A.J., M.C. Okondo, S.D. Rao, K. Gai, A.R. Griswold, D.C. Johnson, D.P. Ball, C.Y. Taabazuing, E.L. Orth, B.A. Vittimberga, and D.A. Bachovchin. 2019. N-terminal degradation activates the NLRP1B inflammasome. Science 364:82-85.

D'Osualdo, A., C.X. Weichenberger, R.N. Wagner, A. Godzik, J. Wooley, and J.C. Reed. 2011. CARD8 and NLRP1 undergo autoproteolytic processing through a ZU5-like domain. PLoS One 6:e27396.

Dixon, A.S., M.K. Schwinn, M.P. Hall, K. Zimmerman, P. Otto, T.H. Lubben, B.L. Butler, B.F. Binkowski, T. Machleidt, T.A. Kirkland, M.G. Wood, C.T. Eggers, L.P. Encell, and K.V. Wood. 
2016. NanoLuc Complementation Reporter Optimized for Accurate Measurement of Protein Interactions in Cells. ACS Chem. Biol. 11:400-408.

Finger, J.N., J.D. Lich, L.C. Dare, M.N. Cook, K.K. Brown, C. Duraiswami, J. Bertin, and P.J. Gough. 2012. Autolytic proteolysis within the function to find domain (FIIND) is required for NLRP1 inflammasome activity. J. Biol. Chem. 287:25030-25037.

Frew, B.C., V.R. Joag, and J. Mogridge. 2012. Proteolytic processing of NIrp1b is required for inflammasome activity. PLoS Pathog. 8:e1002659.

Guey, B., M. Bodnar, S.N. Manie, A. Tardivel, and V. Petrilli. 2014. Caspase-1 autoproteolysis is differentially required for NLRP1b and NLRP3 inflammasome function. Proc. Natl. Acad. Sci. U. S. A. 111:17254-17259.

Hagar, J.A., D.A. Powell, Y. Aachoui, R.K. Ernst, and E.A. Miao. 2013. Cytoplasmic LPS activates caspase-11: implications in TLR4-independent endotoxic shock. Science 341:1250-1253.

He, W.T., H. Wan, L. Hu, P. Chen, X. Wang, Z. Huang, Z.H. Yang, C.Q. Zhong, and J. Han. 2015. Gasdermin $D$ is an executor of pyroptosis and required for interleukin-1beta secretion. Cell Res. 25:1285-1298.

Johnson, D.C., C.Y. Taabazuing, M.C. Okondo, A.J. Chui, S.D. Rao, F.C. Brown, C. Reed, E. Peguero, E. de Stanchina, A. Kentsis, and D.A. Bachovchin. 2018. DPP8/DPP9 inhibitor-induced pyroptosis for treatment of acute myeloid leukemia. Nat. Med. 24:1151-1156.

Jones, J.W., N. Kayagaki, P. Broz, T. Henry, K. Newton, K. O'Rourke, S. Chan, J. Dong, Y. Qu, M. Roose-Girma, V.M. Dixit, and D.M. Monack. 2010. Absent in melanoma 2 is required for innate immune recognition of Francisella tularensis. Proc. Natl. Acad. Sci. U. S. A. 107:9771-9776.

Kayagaki, N., I.B. Stowe, B.L. Lee, K. O'Rourke, K. Anderson, S. Warming, T. Cuellar, B. Haley, M. Roose-Girma, Q.T. Phung, P.S. Liu, J.R. Lill, H. Li, J. Wu, S. Kummerfeld, J. Zhang, W.P. Lee, S.J. Snipas, G.S. Salvesen, L.X. Morris, L. Fitzgerald, Y. Zhang, E.M. Bertram, C.C. Goodnow, and V.M. Dixit. 2015. Caspase-11 cleaves gasdermin D for non-canonical inflammasome signalling. Nature 526:666-671.

Lamkanfi, M., and V.M. Dixit. 2014. Mechanisms and functions of inflammasomes. Cell 157:10131022.

Lee, B.L., I.B. Stowe, A. Gupta, O.S. Kornfeld, M. Roose-Girma, K. Anderson, S. Warming, J. Zhang, W.P. Lee, and N. Kayagaki. 2018. Caspase-11 auto-proteolysis is crucial for noncanonical inflammasome activation. J. Exp. Med. 215:2279-2288.

Mariathasan, S., K. Newton, D.M. Monack, D. Vucic, D.M. French, W.P. Lee, M. Roose-Girma, S. Erickson, and V.M. Dixit. 2004. Differential activation of the inflammasome by caspase-1 adaptors ASC and Ipaf. Nature 430:213-218.

Masters, S.L., M. Gerlic, D. Metcalf, S. Preston, M. Pellegrini, J.A. O'Donnell, K. McArthur, T.M. Baldwin, S. Chevrier, C.J. Nowell, L.H. Cengia, K.J. Henley, J.E. Collinge, D.L. Kastner, L. Feigenbaum, D.J. Hilton, W.S. Alexander, B.T. Kile, and B.A. Croker. 2012. NLRP1 inflammasome activation induces pyroptosis of hematopoietic progenitor cells. Immunity 37:1009-1023.

Okondo, M.C., D.C. Johnson, R. Sridharan, E.B. Go, A.J. Chui, M.S. Wang, S.E. Poplawski, W. Wu, Y. Liu, J.H. Lai, D.G. Sanford, M.O. Arciprete, T.R. Golub, W.W. Bachovchin, and D.A. Bachovchin. 2017. DPP8 and DPP9 inhibition induces pro-caspase-1-dependent monocyte and macrophage pyroptosis. Nat. Chem. Biol. 13:46-53. 
Okondo, M.C., S.D. Rao, C.Y. Taabazuing, A.J. Chui, S.E. Poplawski, D.C. Johnson, and D.A. Bachovchin. 2018. Inhibition of Dpp8/9 Activates the NIrp1b Inflammasome. Cell Chem Biol 25:262-267 e265.

Poyet, J.L., S.M. Srinivasula, M. Tnani, M. Razmara, T. Fernandes-Alnemri, and E.S. Alnemri. 2001. Identification of Ipaf, a human caspase-1-activating protein related to Apaf-1. J. Biol. Chem. 276:28309-28313.

Ross, C., A.H. Chan, J. Von Pein, D. Boucher, and K. Schroder. 2018. Dimerization and autoprocessing induce caspase-11 protease activation within the non-canonical inflammasome. Life Sci Alliance 1:e201800237.

Ruhl, S., K. Shkarina, B. Demarco, R. Heilig, J.C. Santos, and P. Broz. 2018. ESCRT-dependent membrane repair negatively regulates pyroptosis downstream of GSDMD activation. Science 362:956-960.

Sandstrom, A., P.S. Mitchell, L. Goers, E.W. Mu, C.F. Lesser, and R.E. Vance. 2019. Functional degradation: A mechanism of NLRP1 inflammasome activation by diverse pathogen enzymes. Science 364:

Shi, J., Y. Zhao, K. Wang, X. Shi, Y. Wang, H. Huang, Y. Zhuang, T. Cai, F. Wang, and F. Shao. 2015. Cleavage of GSDMD by inflammatory caspases determines pyroptotic cell death. Nature 526:660-665.

Taabazuing, C.Y., M.C. Okondo, and D.A. Bachovchin. 2017. Pyroptosis and Apoptosis Pathways Engage in Bidirectional Crosstalk in Monocytes and Macrophages. Cell Chem Biol 24:507514 e504.

Thornberry, N.A., H.G. Bull, J.R. Calaycay, K.T. Chapman, A.D. Howard, M.J. Kostura, D.K. Miller, S.M. Molineaux, J.R. Weidner, J. Aunins, and et al. 1992. A novel heterodimeric cysteine protease is required for interleukin-1 beta processing in monocytes. Nature 356:768-774.

Van Opdenbosch, N., P. Gurung, L. Vande Walle, A. Fossoul, T.D. Kanneganti, and M. Lamkanfi. 2014. Activation of the NLRP1b inflammasome independently of ASC-mediated caspase1 autoproteolysis and speck formation. Nat Commun 5:3209.

Yang, J., Y. Zhao, and F. Shao. 2015. Non-canonical activation of inflammatory caspases by cytosolic LPS in innate immunity. Curr. Opin. Immunol. 32:78-83.

Zhong, F.L., O. Mamai, L. Sborgi, L. Boussofara, R. Hopkins, K. Robinson, I. Szeverenyi, T. Takeichi, R. Balaji, A. Lau, H. Tye, K. Roy, C. Bonnard, P.J. Ahl, L.A. Jones, P. Baker, L. Lacina, A. Otsuka, P.R. Fournie, F. Malecaze, E.B. Lane, M. Akiyama, K. Kabashima, J.E. Connolly, S.L. Masters, V.J. Soler, S.S. Omar, J.A. McGrath, R. Nedelcu, M. Gribaa, M. Denguezli, A. Saad, S. Hiller, and B. Reversade. 2016. Germline NLRP1 Mutations Cause Skin Inflammatory and Cancer Susceptibility Syndromes via Inflammasome Activation. Cell 167:187-202 e117.

Zhong, F.L., K. Robinson, D.E.T. Teo, K.Y. Tan, C. Lim, C.R. Harapas, C.H. Yu, W.H. Xie, R.M. Sobota, V.B. Au, R. Hopkins, A. D'Osualdo, J.C. Reed, J.E. Connolly, S.L. Masters, and B. Reversade. 2018. Human DPP9 represses NLRP1 inflammasome and protects against autoinflammatory diseases via both peptidase activity and FIIND domain binding. J. Biol. Chem. 293:18864-18878. 


\section{$474 \quad$ Figures}

475

A

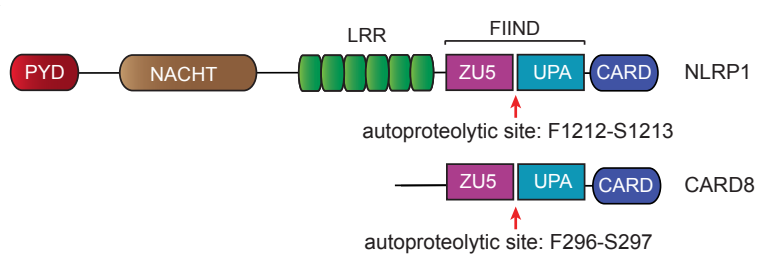

PYD CARD ASC
D

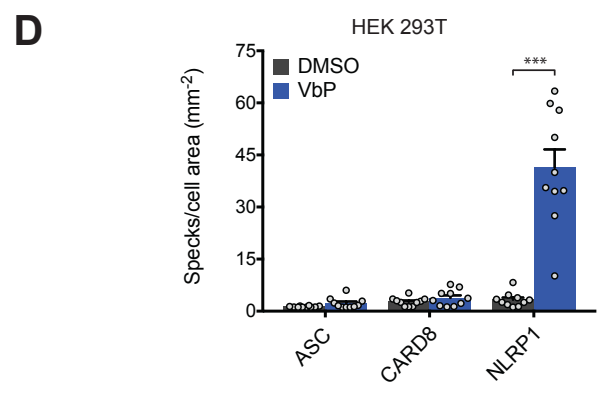

$\mathbf{E}$

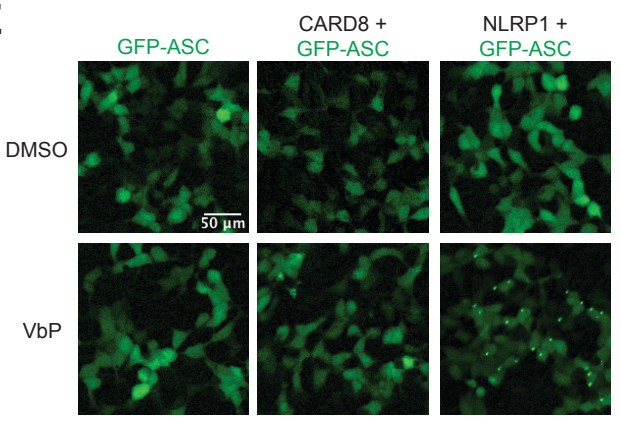

F

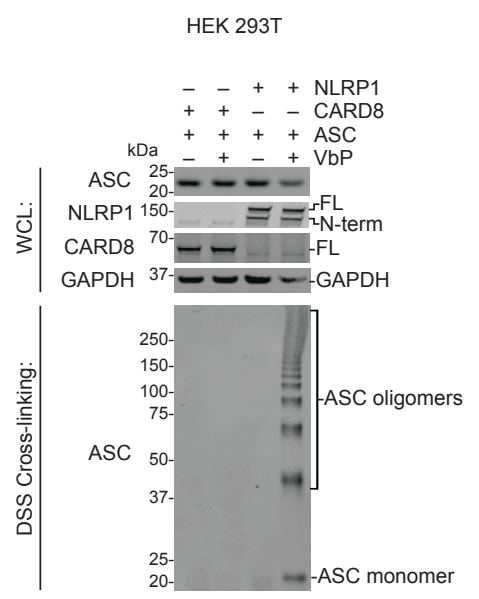

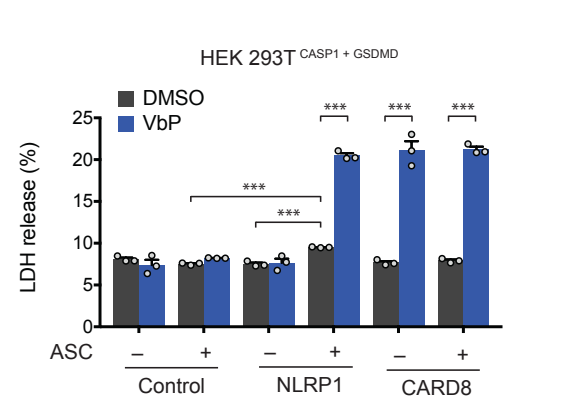

C

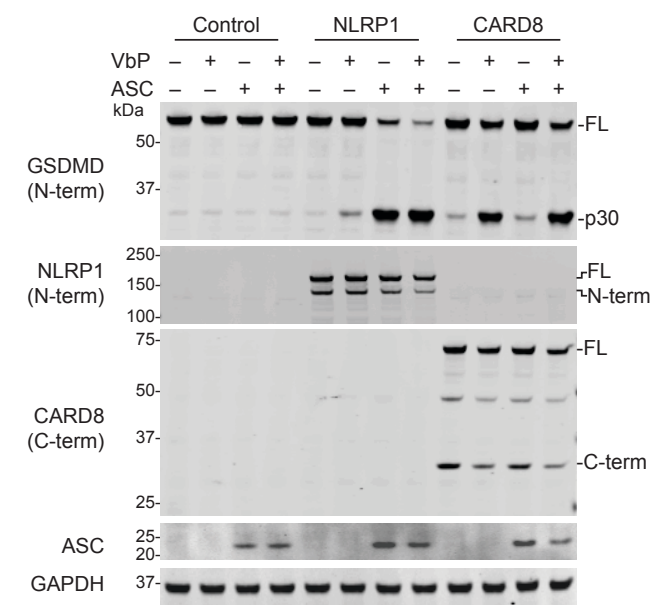

B

477 Figure 1. NLRP1 is ASC-dependent and CARD8 is ASC-independent. (A) Schematic for

478 human NLRP1, CARD8, and ASC protein domain structures. The autoproteolysis sites are

479 indicated. The ZU5-UPA domains together are also referred to as a FIIND. (B,C) HEK 293T cells

480 stably expressing CASP1 and GSDMD (HEK 293T ${ }^{\text {CASP1 + GSDMD }}$ ) were transfected with constructs

481 encoding the indicated proteins and treated with DMSO or VbP (10 $\mu \mathrm{M}, 6 \mathrm{~h})$. Supernatants were

482 evaluated for LDH release (B) and lysates were analyzed by immunoblotting (C). Data are means 
$483 \pm$ SEM of three biological replicates. ${ }^{* * *} p<0.001$ by two-sided Students $t$-test. FL, full-length.

484 (D,E) HEK 293T cells were transfected with constructs encoding GFP-tagged ASC and NLRP1

485 or CARD8, treated with DMSO or $\operatorname{VbP}(10 \mu \mathrm{M}, 6 \mathrm{~h})$, and evaluated for ASC speck formation by

486 fluorescence microscopy. Shown are the mean \pm SEM (D) and representative images (E) from

48710 technical replicates from one of two independent experiments. ${ }^{* *} p<0.001$ by two-sided

488 Students $t$-test. (F) HEK 293T cells transiently transfected with constructs encoding the indicated

489 proteins and treated with DMSO or $\mathrm{VbP}(10 \mu \mathrm{M}, 6 \mathrm{~h})$. Lysates were harvested, subjected to DSS

490 crosslinking, and evaluated by immunoblotting.

491

492

493

494

495

496

497

498

499

500

501

502

503

504

505 
A

507

508

509

510

511

512

513

514

515

516

517

518

519

520

521

522

.

B

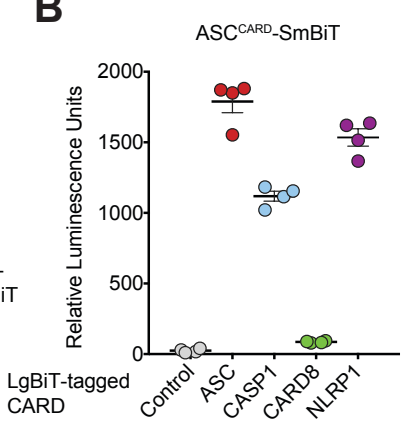

C

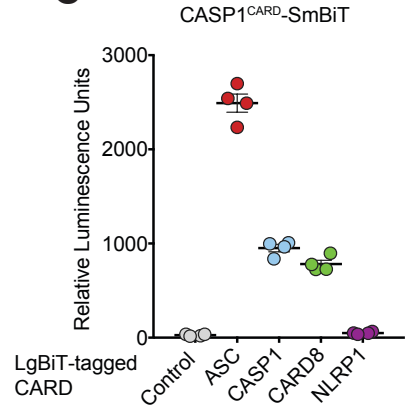

Figure 2. Specific CARD-CARD interactions determine ASC-dependent or independent inflammasome assembly. (A) Expression of the indicated LgBiT-tagged CARDs in HEK 293T cells was verified by immunoblotting. (B,C) Cell lysates from HEK 293T cells transiently expressing LgBiT-tagged ASC ${ }^{\text {CARD }}$ (B) or LgBiT-tagged CASP1 ${ }^{\text {CARD }}$ (C) were mixed with lysates containing SmBiT-tagged CARDs and analyzed for the relative luminescence.

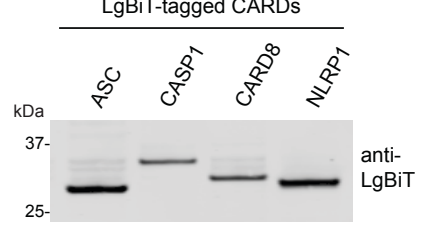


A

HEK 293T'AASP1 + asono

DMSO

$\mathrm{VbP}$

$\mathrm{VbP}+$ bortezomib

$\mathrm{VbP}+\mathrm{MG} 132$

$\mathrm{VbP}+$ carfilzomib

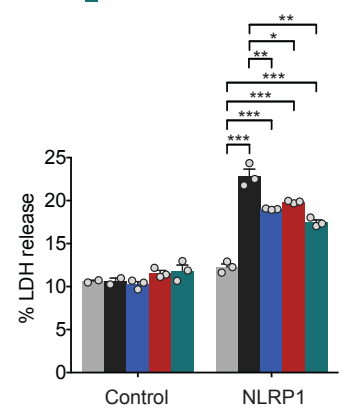

B

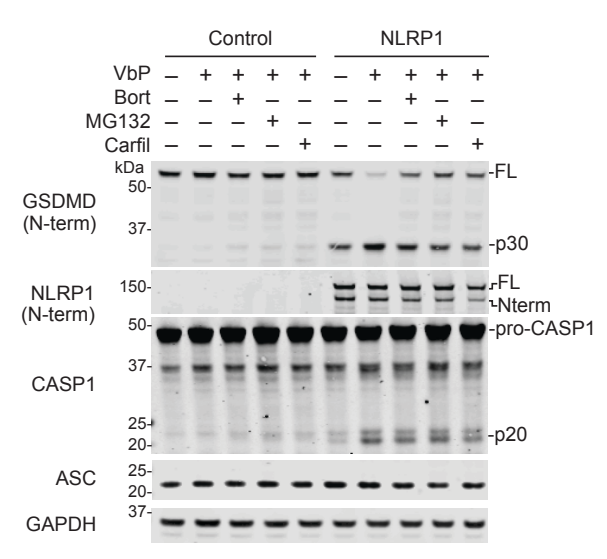

C

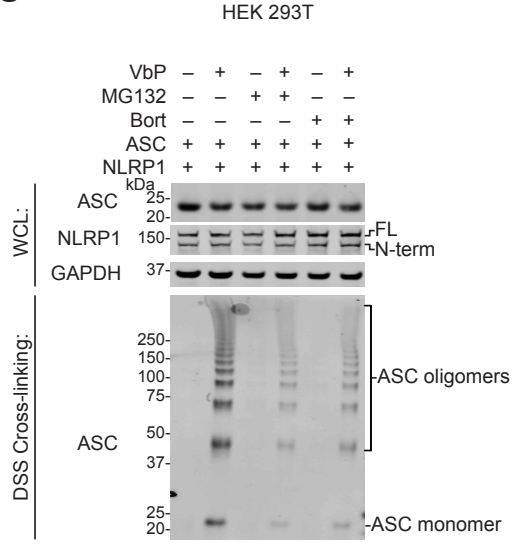

524 Figure 3. Proteasome inhibitors block NLRP1 inflammasome activation. (A,B) HEK

$525293 T^{\text {CASP1 + GSDMD }}$ were transiently transfected with constructs encoding NLRP1 and ASC,

526 pretreated with the indicated proteasome inhibitors $(20 \mu \mathrm{M}, 30 \mathrm{~min})$, and stimulated with $\mathrm{VbP}(10$

$527 \mu \mathrm{M}, 6 \mathrm{~h})$. Supernatants were evaluated for LDH release (B) and lysates were analyzed by

528 immunoblotting (C). Data are means \pm SEM of three biological replicates and representative of

529 two independent experiments. ${ }^{*} p<0.05^{*},{ }^{*} p<0.01,{ }^{* * *} p<0.001$ by two-sided Students $t$-test.

530 (C) HEK 293T cells transiently transfected with constructs encoding NLRP1 and ASC,

531 preincubated with MG132, carfilzomib, or bortezomib (20 $\mu \mathrm{M}, 30 \mathrm{~min})$, and treated with DMSO or

$532 \operatorname{VbP}(10 \mu \mathrm{M}, 6 \mathrm{~h})$.

533

534

535

536

537

538

539

540

541 
A

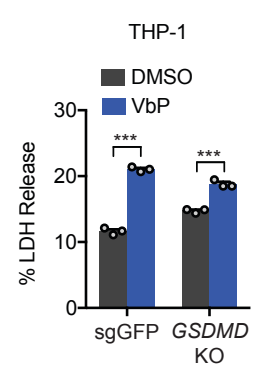

B

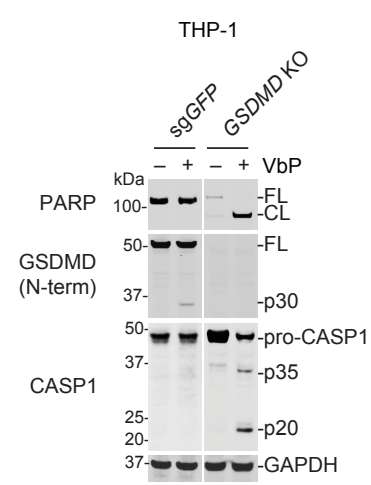

C

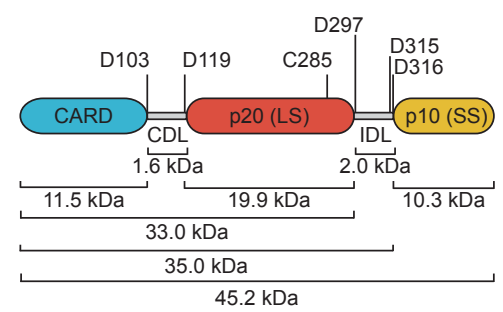

D

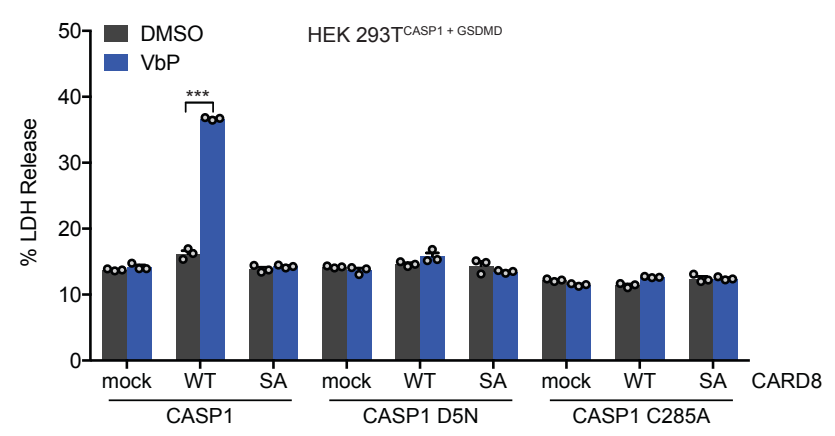

E

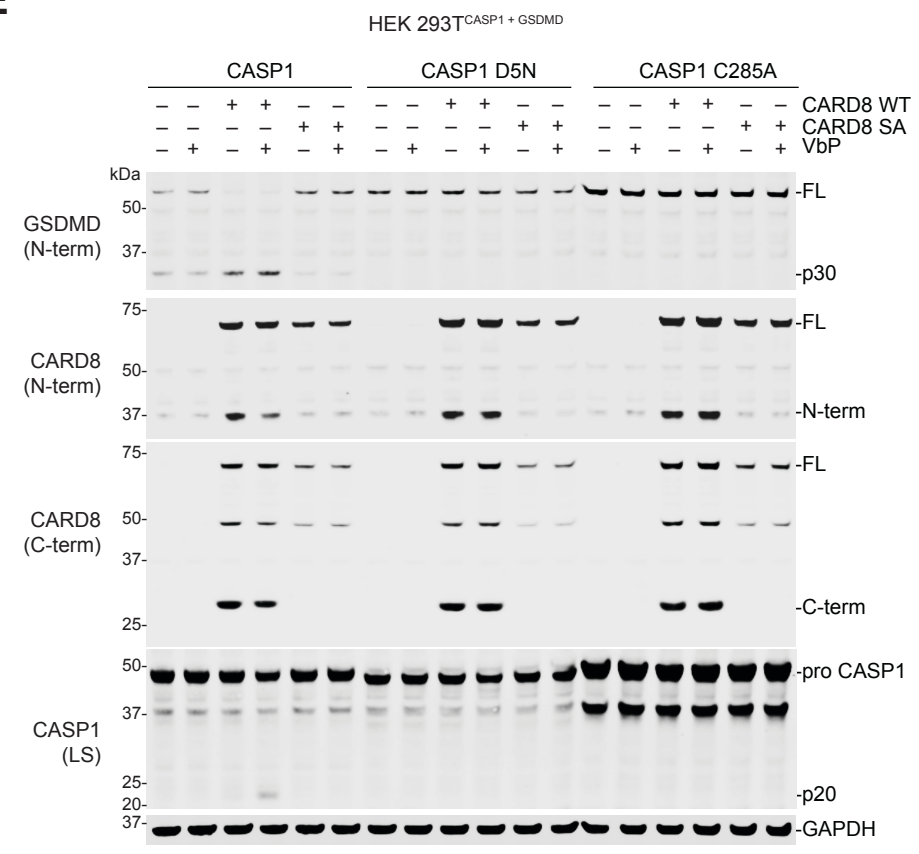

543 Figure 4. Caspase-1 autoproteolysis is required for CARD8 inflammasome activation. (A,B)

544 Control and GSDMD ${ }^{-/}$THP-1 cells were treated with $\mathrm{VbP}(10 \mu \mathrm{M}, 24 \mathrm{~h})$ before supernatants were

545 analyzed for LDH release (A) and lysates were evaluated by immunoblotting (B). Data are means

$546 \pm$ SEM of three biological replicates. ${ }^{* * *} p<0.001$ by two-sided Students $t$-test. FL, full-length.

$547 \mathrm{CL}$, cleaved. (C) Schematic of pro-caspase-1 depicting the CARD domain and large (p20, LS)

548 and small $(\mathrm{p} 10, \mathrm{SS})$ catalytic subunits. Predicted cleavage sites, sizes of potential cleavage

549 products, and the catalytic cysteine are indicated. (D, E) HEK 293T cells stably expressing

550 GSDMD and the indicated pro-caspase-1 constructs were transiently transfected with plasmids

551 encoding RFP (mock), CARD8 WT, or autoproteolysis-defective CARD8 S297A (SA) for $24 \mathrm{~h}$

552 before addition of $\mathrm{VbP}(10 \mu \mathrm{M}, 6 \mathrm{~h})$. Cell death was assessed by LDH release (D) and GSDMD 
553 and CASP1 cleavage by immunoblotting (E). Data are means \pm SEM of three biological

554 replicates. ${ }^{* * *} p<0.001$ by two-sided Students $t$-test.

555

556

557

558

559

560

561

562

563

564

565

566

567

568

569

570 
A
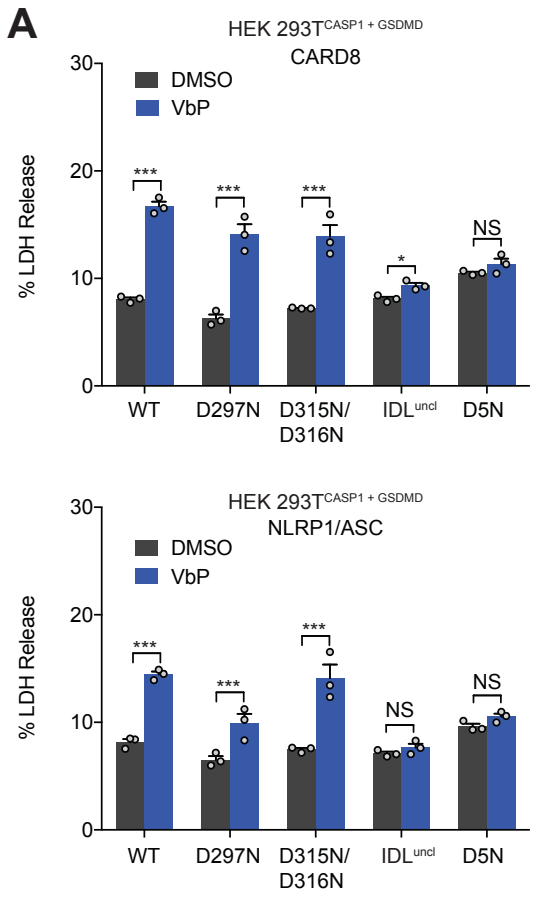

B HEK 293T 2 CASP + + GSOMD
NLRP $1 /$ ASC
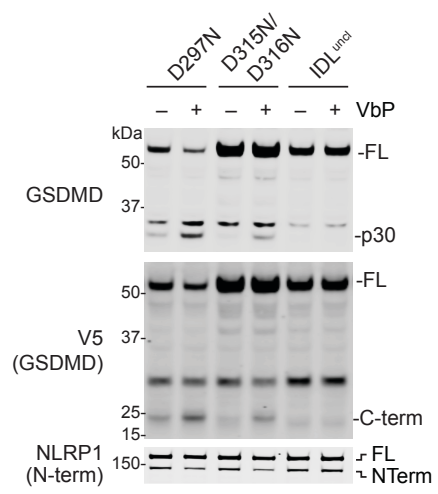

50-ヒUルルヒー- pro CASP1 CASP1

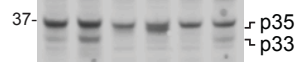
25

ASC

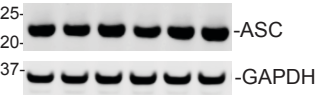

C

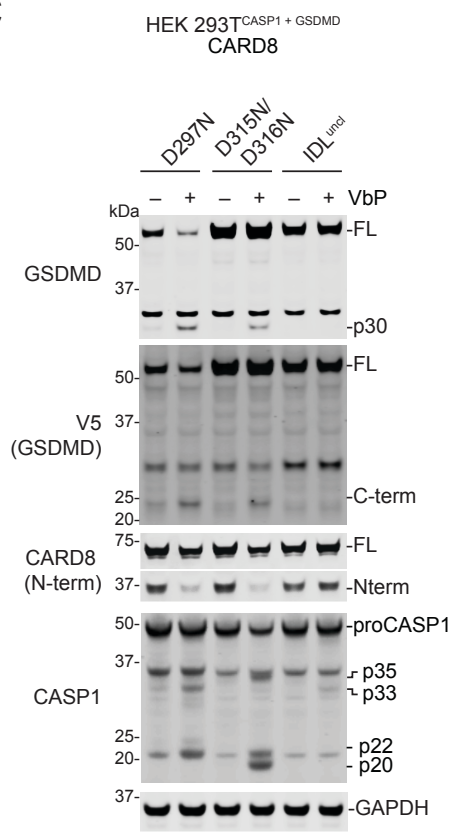

574 Figure 5. Cleavage of the human caspase-1 IDL is required for activation of canonical

575 inflammasomes. (A-C) HEK 293T cells stably expressing GSDMD and the indicated pro576 caspase-1 constructs were transiently transfected with plasmids encoding NLRP1 $(0.1 \mu \mathrm{g})$ and

577 ASC $(0.01 \mu \mathrm{g})(\mathbf{A}, \mathbf{B})$ or CARD8 $(\mathbf{A}, \mathbf{C})$ for $24 \mathrm{~h}$ before addition of $\operatorname{VbP}(10 \mu \mathrm{M}, 6 \mathrm{~h})$. Cell death 578 was assessed by $\mathrm{LDH}$ release $(\mathbf{A})$ and GSDMD cleavage by immunoblotting $(\mathbf{B}, \mathbf{C})$. Data are 579 means \pm SEM of three biological replicates. ${ }^{*} p<0.05,{ }^{* * *} p<0.001$ by two-sided Students $t$ 580 test. NS, not significant. 

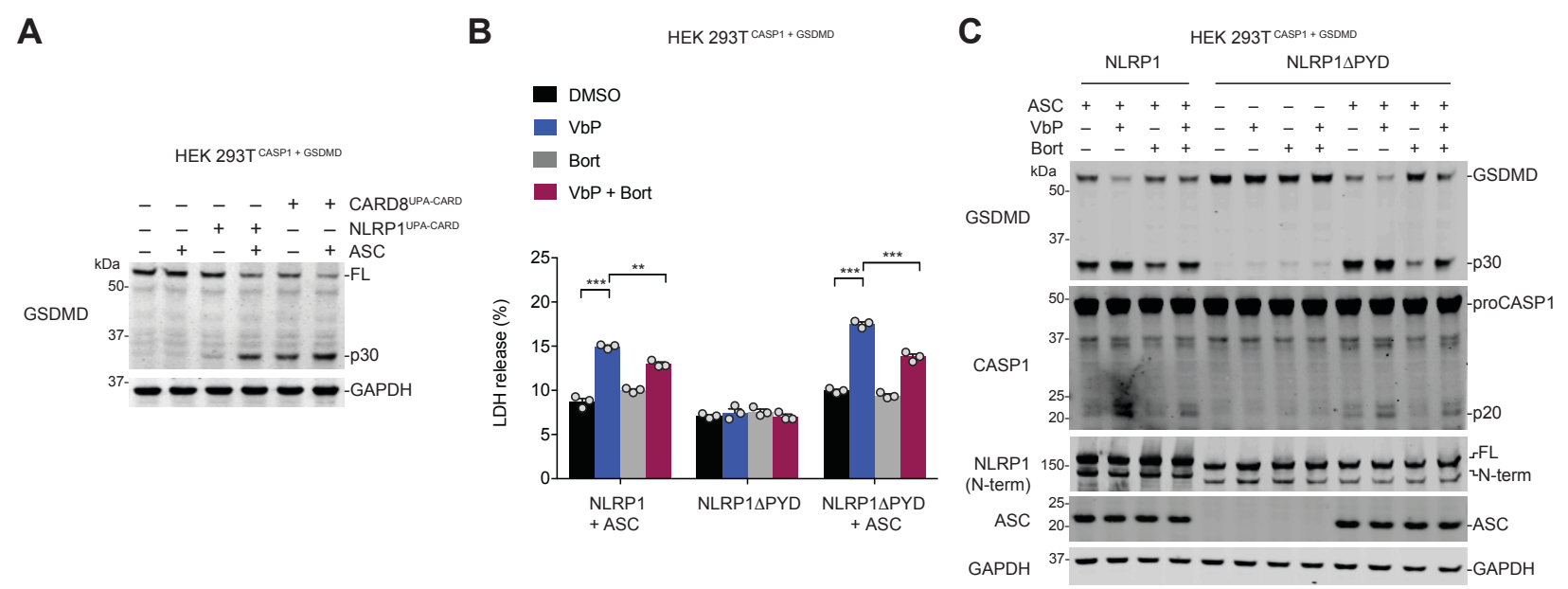

D

E

HEK 293T
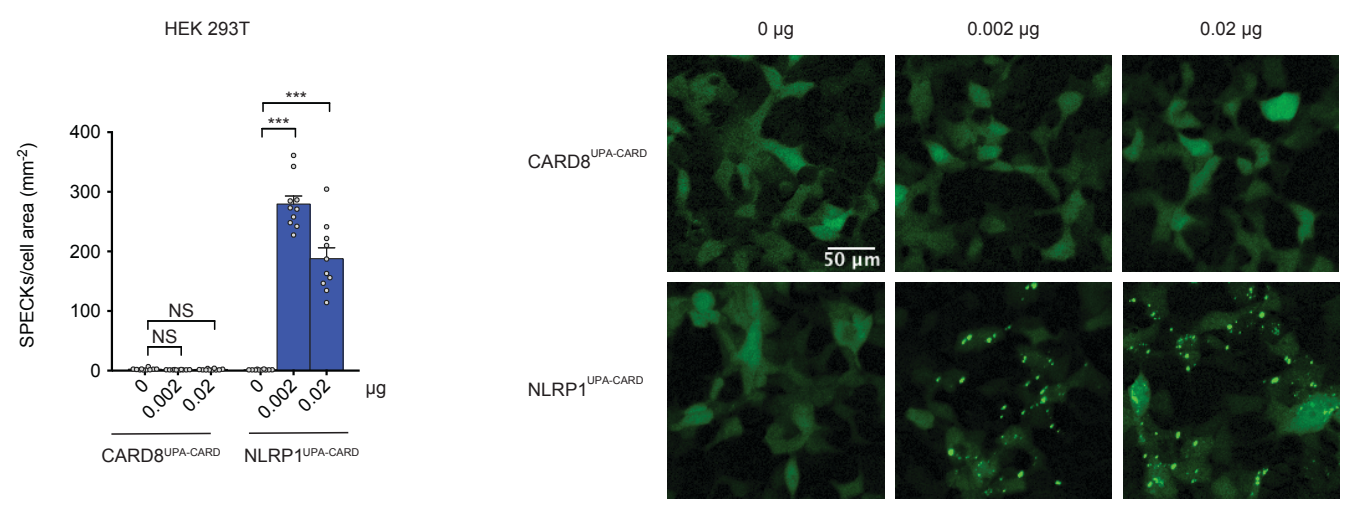

Figure S1. The NLRP1 CARD is responsible for inflammasome activation. (A) HEK 293T ${ }^{\text {CASP1 }}$

+ GSDMD cells were transfected with plasmids encoding the UPA-CARD fragments of NLRP1 or

587 CARD8 and ASC as indicated. After 24 h, lysates were evaluated by immunoblotting. (B,C) HEK

$588293 T^{\text {CASP1 + GSDMD }}$ were transfected with plasmids encoding full-length NLRP1 or NLRP1 without a

pyrin domain (NLRP1 $\triangle P Y D)$ and treated with DMSO or VbP $(10 \mu \mathrm{M}, 6 \mathrm{~h})$. Supernatants were

$591 \pm$ SEM of three biological replicates. ${ }^{* *} p<0.01,{ }^{* * *} p<0.001$ by two-sided Students $t$-test. $(\mathrm{D}, \mathrm{E})$

592 HEK 293T cells were transfected with plasmids encoding GFP-tagged ASC and the UPA-CARD

593 fragments of NLRP1 or CARD8, and then evaluated for ASC speck formation by fluorescence 
594 microscopy. Shown are the mean \pm SEM (D) and representative images (E) from 10 technical

595 replicates from one of two independent experiments. ${ }^{* * *} p<0.001$, by two-sided Students $t$-test.

596 NS, not significant.

597

598

599

600

601

602

603

604

605

606

607

608

609 


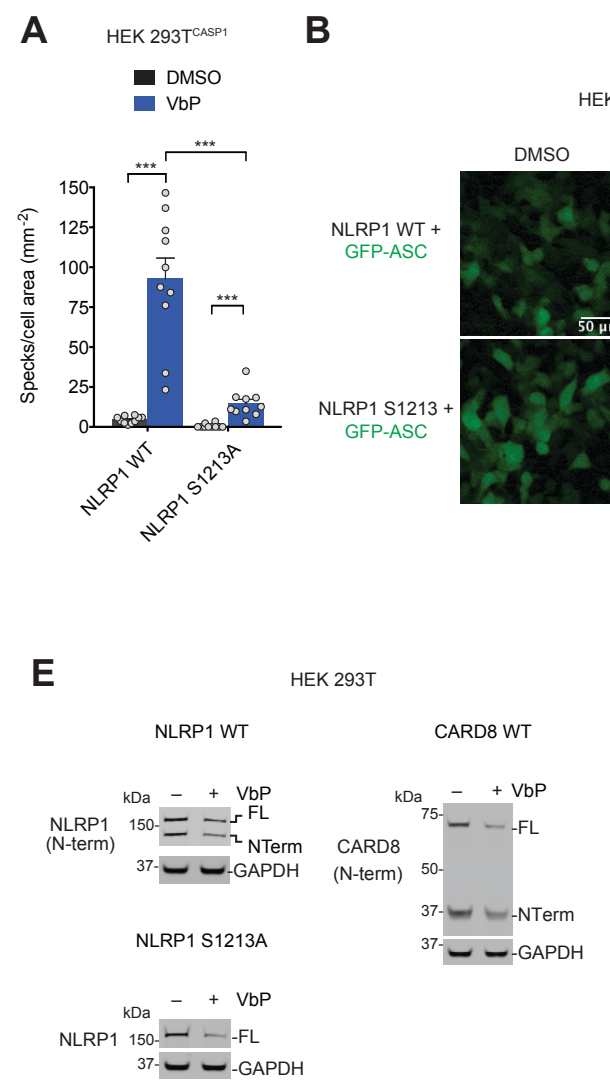

610
C

HEK 293T CASP1+ GSDMD
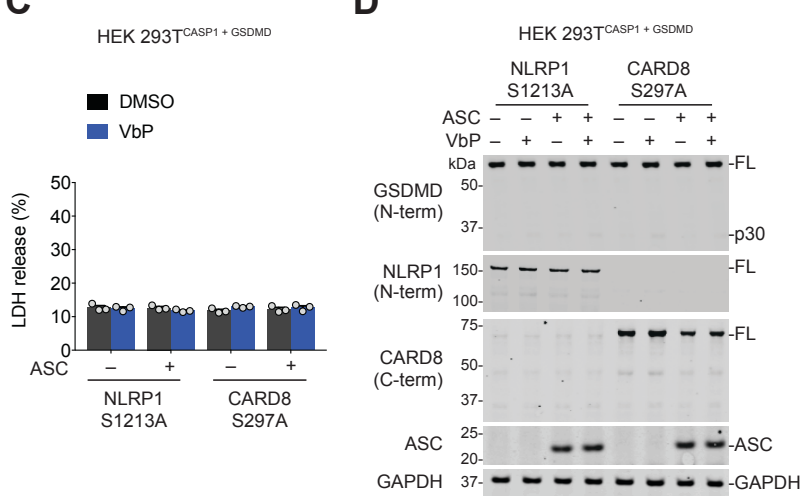

F

HEK 293T CASP1+ GSDMD

G

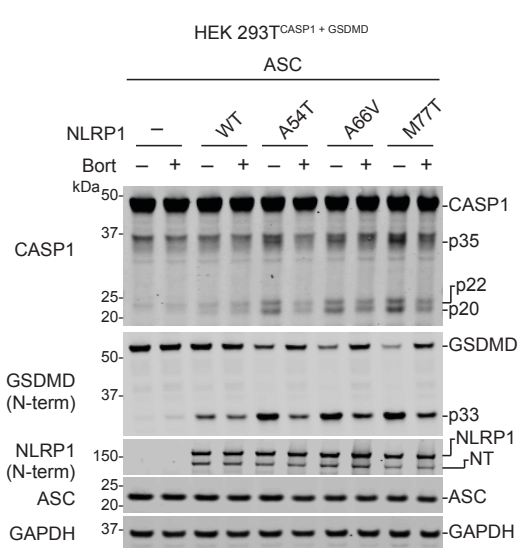

Figure S2. VbP and germline mutations activate NLRP1 via N-terminal degradation. (A,B) HEK 293T cells stably expressing CASP1 were transfected with plasmids encoding GFP-tagged ASC and the indicated NLRP1 protein, treated with DMSO or VbP (10 $\mu \mathrm{M}, 6 \mathrm{~h})$, and evaluated for ASC speck formation by fluorescence microscopy. Shown are the mean \pm SEM (A) and representative images (B) from 10 technical replicates from one of two independent experiments. ${ }^{* * *} p<0.001$, by two-sided Students $t$-test. (C,D) HEK 293T CASP1 + GSDMD cells were transiently transfected with plasmids encoding autoproteolytic cleavage-deficient NLRP1 S1213A or CARD8 S297A with and without ASC, treated with $\mathrm{VbP}(10 \mu \mathrm{M}, 6 \mathrm{~h})$, and evaluated for LDH release (C) and GSDMD cleavage by immunoblotting (D). The data are means \pm SEM of three biological replicates. (E) HEK 293T cells were transiently transfected with the plasmids encoding CARD8 WT $(0.1 \mu \mathrm{g})$, NLRP1 WT $(0.5 \mu \mathrm{g})$, or NLRP1 S1213A $(0.02 \mu \mathrm{g})$ prior to treatment with VbP $(10$ 
$623 \mu \mathrm{M}, 24 \mathrm{~h})$. Cells were then treated with $\mathrm{VbP}$ again $(10 \mu \mathrm{M}, 24 \mathrm{~h}-$ total of $48 \mathrm{~h}$ of treatment)

624 before lysates were evaluated by immunoblotting. (F,G) HEK $293 T^{\text {CASP1 + GSDMD }}$ cells were

625 transiently transfected with plasmids encoding the indicated NLRP1 protein and ASC for $24 \mathrm{~h}$

626 before being treated with bortezomib $(20 \mu \mathrm{M}, 6 \mathrm{~h})$. Cell death was evaluated by LDH release (F)

627 and lysates assessed by immunoblotting (G). Data are means \pm SEM of three biological

628 replicates. ${ }^{* *} p<0.01,{ }^{* * *} p<0.001$ by two-sided Students $t$-test.

629

630

631

632

633

634

635

636

637

638

639

640

641

642

643

644

645

646

647

648 
A

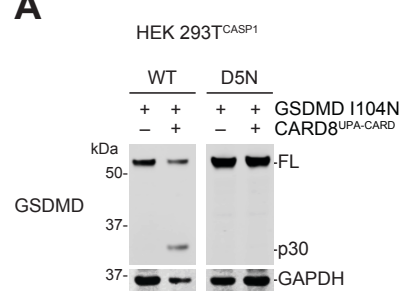

D

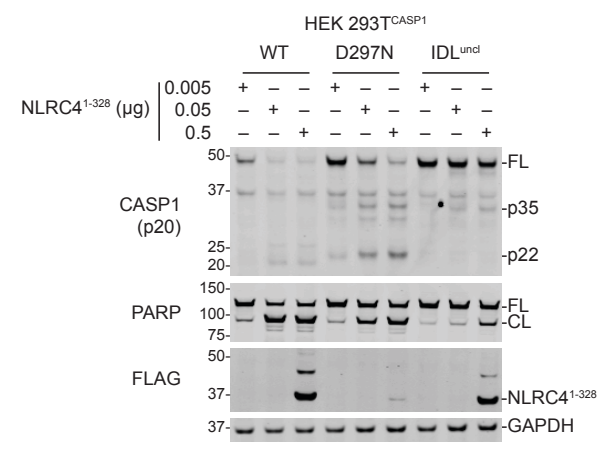

649

650

651

652

653

654

655
B

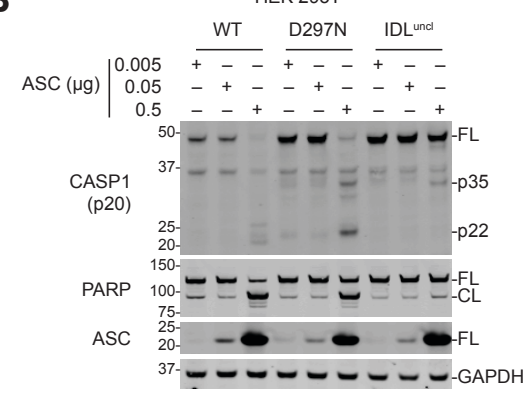

E
C

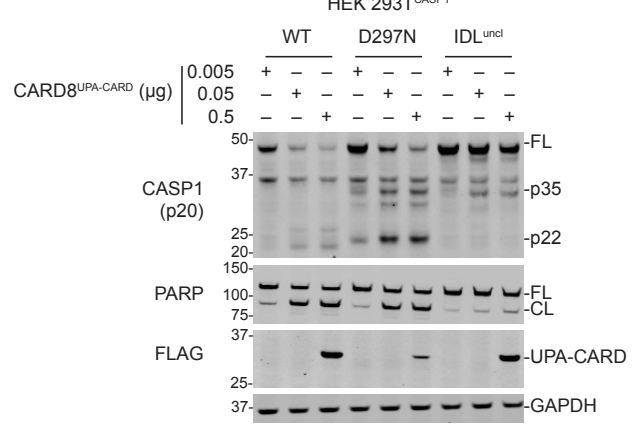

$\mathbf{F}$

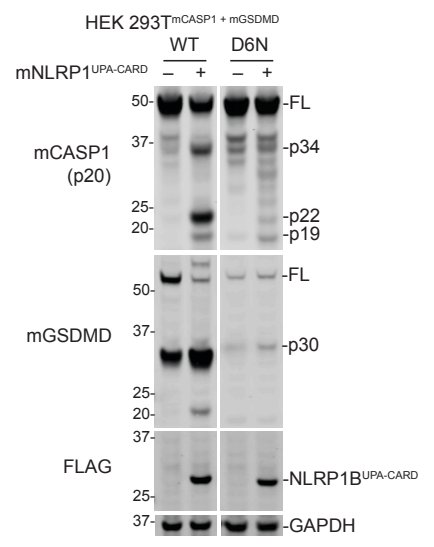

G

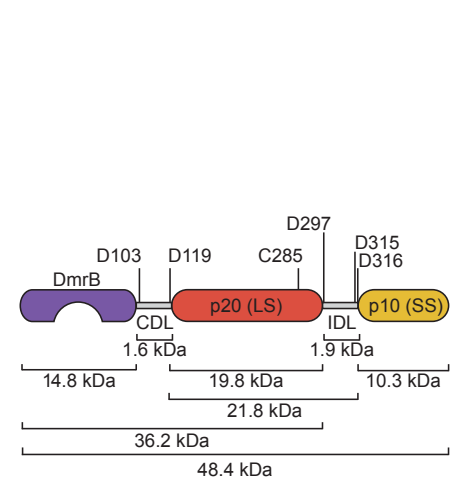

H

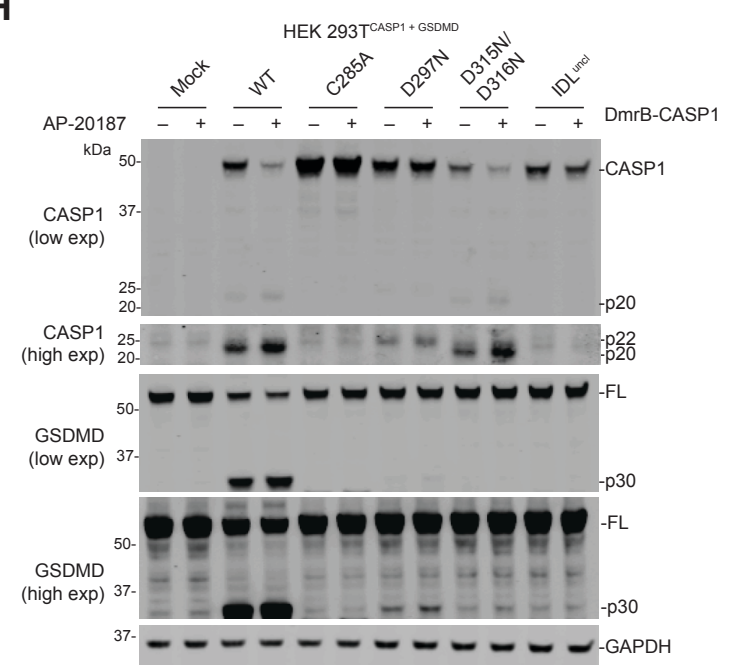

Figure S3. IDL cleavage is necessary for human pro-caspase-1 activation. (A) HEK 293T cells stably expressing CASP1 WT or CASP1 D5N were transiently transfected with plasmids encoding the UPA-CARD fragment of CARD8 $(0.1 \mu \mathrm{g})$ and GSDMD I104N $(0.1 \mu \mathrm{g})$. After $24 \mathrm{~h}$, lysates were evaluated by immunoblotting. (B-D) HEK 293T cells stably expressing the indicated pro-caspase-1 were transiently transfected with the indicated amounts of plasmids encoding ASC (B) the UPA-CARD of CARD8 (C), or residues 1-328 of NLRC4 (D). Lysates were evaluated by 
656 immunoblotting after 24 h. (E, F) HEK 293T cells stably expressing mouse CASP1 WT or CASP1

657 D6N and mouse GSDMD were transiently transfected with a plasmid encoding the UPA-CARD

658 fragment of NLRP1B $(0.1 \mu \mathrm{g})$. After $24 \mathrm{~h}$, supernatants were assessed for LDH release $(\mathrm{E})$ and

659 lysates were evaluated by immunoblotting (F). Data are means \pm SEM of two or three biological

660 replicates. ${ }^{* * *} p<0.001$ by two-sided Students $t$-test. (G) Schematic of the DmrB-caspase-1

661 constructs. Predicted cleavage sites, sizes of potential cleavage products, and the catalytic

662 cysteine are indicated. (H) HEK 293T cells stably expressing GSDMD were transiently transfected

663 with the indicated DmrB-caspase-1 constructs for $24 \mathrm{~h}$ before addition of AP-20187 (500 nM, 1

664 h). GSDMD and CASP1 cleavage were evaluated by immunoblotting.

665

666

667

668

669

670 